\title{
Dependency of Conductive Atomic Force Microscopy and Lateral Force Microscopy Signals on Scan Parameters for Zinc Oxide Nanorods
}

\author{
Yijun Yang and Kwanlae Kim* \\ Department of Manufacturing Systems and Design Engineering (MSDE), Seoul National University of \\ Science and Technology (SeoulTech), Seoul 01811, Republic of Korea
}

\begin{abstract}
Conductive atomic force microscopy (C-AFM) is one of the most commonly used characterization techniques for piezoelectric one-dimensional nanomaterials. However, a comprehensive understanding of the effects of certain scan parameters on the C-AFM signals remains elusive. In the present work, the dependency of C-AFM signals on the normal force, scan speed, and Z scanner feedback gain was studied in conjunction with lateral force microscopy (LFM) signals. As the normal force increased, the C-AFM and the LFM signals increased for the following two possible reasons. When larger normal force was applied, $\mathrm{ZnO}$ nanorods were more effectively deflected, intensifying the piezoelectric effect. Additionally, the triboelectric effect was enhanced via the increased force of friction between the AFM tip and the ZnO nanorods. When the scan speed increased to $0.5 \mathrm{~Hz}$, the LFM signals and the C-AFM signals increased owing to the enhanced degree of deflection in the $\mathrm{ZnO}$ nanorods. However, when exceeding $0.5 \mathrm{~Hz}$, both the LFM signals and the C-AFM signals started to decrease because the AFM tip did not come into contact with the short ZnO nanorods at a high scan speed. Finally, with an increase in the feedback gain to 0.5, both the LFM signals and the CAFM signals increased. However, when the feedback gain exceeded 1.0, the $\mathrm{Z}$ scanner feedback loop was too sensitive to deflect the $\mathrm{ZnO}$ nanorod, considerably reducing the total LFM signals. In contrast, the total CAFM signal showed only a moderate decrease.
\end{abstract}

(Received September 7 2021; Accepted November 4, 2021)

Keywords: $\mathrm{ZnO}$ nanorods, conductive AFM, lateral force microscopy, scan parameters, piezoelectric, triboelectric

\section{Introduction}

Piezoelectric energy harvesting technology has garnered much attention given that it enables self-powering electronics [1]. In particular, wasted mechanical energy during human motion can be converted into electrical energy, implying that an energy harvesting system requires both excellent piezoelectric performance and flexibility [2,3]. Thus, polymers such as poly(vinylidene fluoride) (PVDF) and polydimethylsiloxane (PDMS) are employed with piezoelectric nanomaterials to meet these requirements [4-7]. At present, intensive research on piezoelectric polymers such as PVDF and its copolymers has been conducted, but the piezoelectric coefficients of these polymers are still far below those of

- 양이준: 석사후연구원, 김관래: 교수

*Corresponding Author: Kwanlae Kim

[Tel: +82-2-970-7285, E-mail: klkim@seoultech.ac.kr]

Copyright (c) The Korean Institute of Metals and Materials inorganic piezoelectric nanomaterials [8-10]. Therefore, improving measurement techniques applicable to these nanomaterials is essential to advance the development of piezoelectric energy harvesting systems.

Because $\mathrm{ZnO}$ simultaneously exhibits semiconducting and piezoelectric properties $[11,12]$, intensive research has been carried out related to its application to diverse electronics, including optoelectronic $[13,14]$ and piezoelectric devices $[15,16]$. Piezoelectricity in a $\mathrm{ZnO}$ nanorod was discovered for the first time using conductive atomic force microscopy (C-AFM) [17]. When an AFM probe scans vertically grown $\mathrm{ZnO}$ nanorods in contact mode, the AFM tip deflects the nanorods, generating piezoelectric potential. For n-type $\mathrm{ZnO}$, a Schottky barrier arises between the AFM tip and $\mathrm{ZnO}$ nanorods when the AFM tip touches the compressed side of a $\mathrm{ZnO}$ nanorod, enabling current to flow through the AFM probe and the external circuit [18]. Since this discovery, CAFM has been applied to a wide range of piezoelectric 
nanomaterials. For example, an electric charge flow was detected from p-type $\mathrm{ZnO}$ nanorods when the AFM tip was in contact with the stretched side of a $\mathrm{ZnO}$ nanorod [19]. Subsequently, current measurements using C-AFM were successfully applied to other piezoelectric nanomaterials, including $\mathrm{Pb}(\mathrm{Zr}, \mathrm{Ti}) \mathrm{O}_{3}$ [20], and materials with wurtzite structures [21-25]. Meanwhile, our understanding of C-AFM measurements on $\mathrm{ZnO}$ nanorods has been improved remarkably via advanced characterization techniques. For instance, an C-AFM instrument was integrated with a scanning electron microscope to enable in-situ observations during current measurements of $\mathrm{ZnO}$ nanorods [26]. From this work, it was suggested that the current signals originate from the triboelectric effect between the AFM tip and $\mathrm{ZnO}$ nanorods as well as the piezoelectric potential. This argument is supported by a subsequent study based on simultaneous measurements by C-AFM and lateral force microscopy (LFM) by Yang and Kim [27]. In this more recent work, it was revealed that a considerable amount of current can originate from the triboelectric effect between the AFM tip and the $\mathrm{ZnO}$ nanorod when large normal force is applied with an AFM probe with a large spring constant.

In AFM and AFM-based characterization techniques, the signals are influenced by the scan parameters [28-31]. During contact-mode AFM measurements, topography signals are highly dependent on the scan conditions due to the mechanical interaction between the AFM probe and the sample surface. Thus, when the AFM probe scans freestanding $\mathrm{ZnO}$ nanorods in contact mode, the resultant current map would be influenced considerably by scan parameters such as the normal force, scan speed, and Z scanner feedback gain. For p-type $\mathrm{ZnO}$ nanorods, the effects of scan parameters such as the scan rate and normal force on the number of voltage peaks and the average voltage peak have been investigated [19]. The results showed that the number of voltage peaks increased with an increase in the scan rate and the normal force. In contrast, the average voltage peak did not show a steady increasing trend as the scan rate or normal force increased. However, in spite of this early attempt to understand the relationship between scan parameters and CAFM measurements, a comprehensive understanding of the effects of scan parameters on the physical interaction between the AFM tip and $\mathrm{ZnO}$ nanorods is still elusive.
In the present work, the effects of scan parameters on $\mathrm{C}$ AFM measurements are investigated with regard to freestanding $\mathrm{ZnO}$ nanorods. In particular, C-AFM and LFM are simultaneously employed to analyze the interaction between the AFM tip and $\mathrm{ZnO}$ nanorods systematically. Depending on the normal force, scan speed, and $\mathrm{Z}$ scanner feedback gain, the interaction between the AFM tip and $\mathrm{ZnO}$ nanorods is analyzed using scatter plots of the current versus the lateral force. We show that the variation of the C-AFM signal in response to varying scan parameters is highly dependent on the variation of the LFM signal. The C-AFM signal gradually increases with an increase in the normal force, but the maximum C-AFM and LFM signals could be obtained from the optimal scan speed and the $\mathrm{Z}$ scanner feedback gain.

\section{Experimental Methods}

$\mathrm{ZnO}$ nanorods were grown via a two-step hydrothermal growth method [27]. First, a $p$-Si (100) substrate was sonicated with acetone, ethanol, and deionized (DI) water each for 10 minutes in turn. A seed layer was formed on top of the substrate using zinc acetate dehydrate $\left(\mathrm{Zn}\left(\mathrm{Ch}_{3} \mathrm{COO}\right)_{2}\right.$. $\left.2 \mathrm{H}_{2} \mathrm{O}\right)$ dissolved in ethanol $(50 \mathrm{mM})$ at $80^{\circ} \mathrm{C}$ for $30 \mathrm{~s}$. The seed layer was dried using a hot plate at $100^{\circ} \mathrm{C}$ for five minutes. This growth sequence was repeated three times. Thereafter, $\mathrm{ZnO}$ nanorods were grown on top of the seed layer at $80{ }^{\circ} \mathrm{C}$ for 25 hours using a precursor solution composed of $50 \mathrm{mM}$ zinc nitrate hexahydrate $\left(\mathrm{Zn}\left(\mathrm{NO}_{3}\right)_{2}\right.$. $\left.6 \mathrm{H}_{2} \mathrm{O}\right)$ and hexamethylenetetramine $\left(\left(\mathrm{CH}_{2}\right)_{6} \mathrm{~N}_{4}\right)$ dissolved in DI water.

The morphology of the $\mathrm{ZnO}$ nanorods was characterized using field emission scanning electron microscopy (FE-SEM, JSM6700F, JEOL). The crystal structure of the $\mathrm{ZnO}$ nanorods was analyzed by means of X-ray diffraction (XRD, DE/D8 Advance, Bruker) [32] with $\mathrm{Cu}-\mathrm{K} \alpha$ radiation. $\mathrm{C}-\mathrm{AFM}$ and LFM [33] were simultaneously conducted using an AFM instrument (XE-150, Park Systems). To enable the C-AFM measurements, a C-AFM module and a current amplifier were additionally installed in the AFM instrument. An AFM probe, the PPP-NCHPt type (NanoSensor), with a $42 \mathrm{~N} / \mathrm{m}$ spring constant was used for both the C-AFM and LFM measurements. In the previous study [27], the current originating from triboelectric effect of the AFM tip and $\mathrm{ZnO}$ 
nanorods was clearly observed with PPP-NCHPt. Therefore, the same AFM probe was used to study the effect of scan parameters on the current induced by both piezoelectric and triboelectric effects in response to varying scan parameters. In this work, the scan parameters of the normal force (set point in contact mode), scan speed, and $\mathrm{Z}$ scanner feedback gain were varied to study the effects of the scan parameters on C-AFM and LFM results. The orientation of the cantilever was consistently perpendicular with respect to the fast scan direction.

In this experimental setup, three distinct signals (topography, LFM, and C-AFM) were simultaneously generated, as shown in Fig. 1(a). The dynamic motion of an AFM cantilever is monitored by a laser beam reflected from the cantilever onto a position-sensitive photodetector (PSPD). The C-AFM signals are purely based on the measured current through the conductive AFM tip and a current amplifier. The current can be produced by contact potential, triboelectric, and piezoelectric effects, as illustrated in Fig. 1(b). Earlier work [27] presents additional details with regard to the mechanisms of current generation from $\mathrm{ZnO}$ nanorods by an AFM probe.

$\mathrm{C}$-AFM and LFM signals can be represented correspondingly as $\mathrm{C}-\mathrm{AFM}_{\mathrm{i}, \mathrm{j}}$ and $\mathrm{LFM}_{i, j}$ based on matrix notation. C-AFM signals produced from n-type $\mathrm{ZnO}$ nanorods are negative. Accordingly, $\left|\mathrm{C}-\mathrm{AFM}_{i, j}\right|$ was used for the computation and graphical representations. All data introduced in this work were obtained during trace scans, and LFM signals during trace scan were positive.

(a)

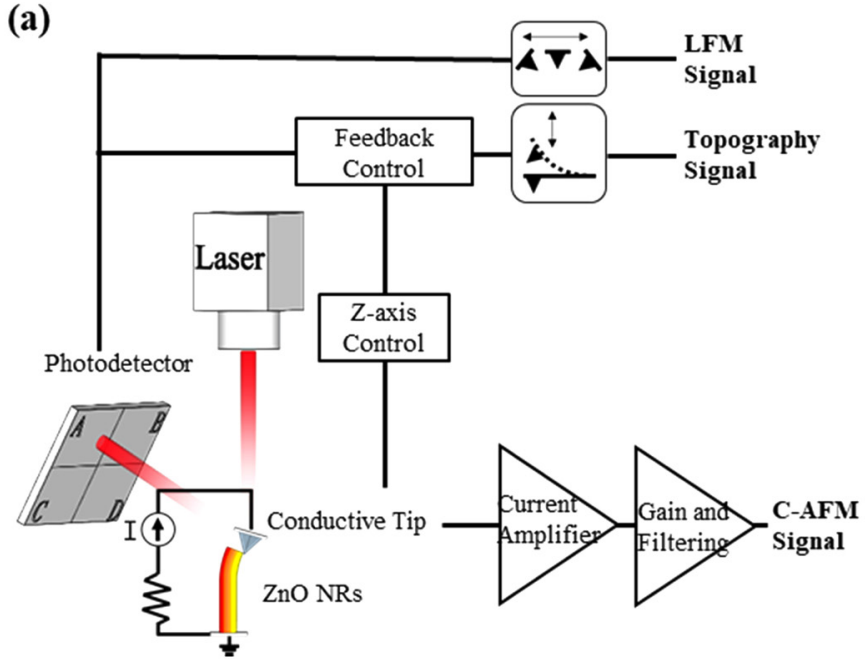

\section{Results and discussion}

The morphology and sizes of the grown $\mathrm{ZnO}$ nanorods were identified using top- and side-view SEM images, as shown in Figs. 2(a)-(b). The measured lengths of the $\mathrm{ZnO}$ nanorods were approximately $2 \mu \mathrm{m}$. From Fig. 2(c), a hexagonal crystal system in $\mathrm{ZnO}$ nanorods can be identified by the (100), (002), and (101) peaks. It is confirmed that $\mathrm{ZnO}$ nanorods were successfully grown on the $\mathrm{Si}$ substrate, and the observed morphology is suitable for C-AFM and LFM (a)

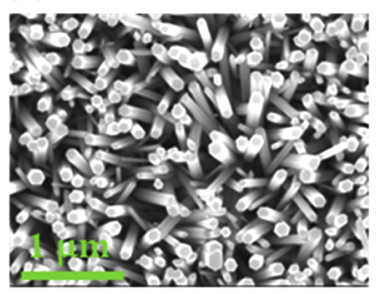

(b)

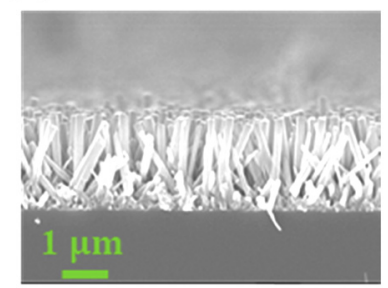

(c)

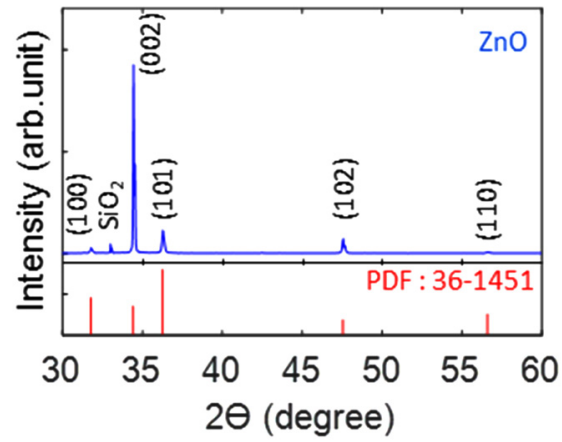

Fig. 2. (a) Top-view and (b) side-view images of vertically grown $\mathrm{ZnO}$ nanorods, and (c) XRD profiles of the $\mathrm{ZnO}$ nanorods.

(b)

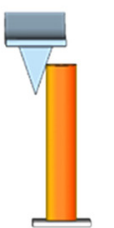

Contact potential

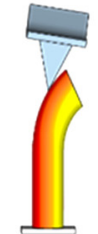

Triboelectric

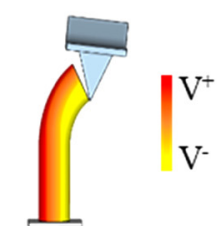

Triboelectric

\&
Piezoelectric

Fig. 1. (a) Schematic the illustrating simultaneous acquisition of C-AFM and LFM signals, (b) three distinct current-generating mechanisms: contact potential, triboelectricity, and piezoelectricity. 
measurements.

To study the effects of normal force on the C-AFM and LFM signals, $300-600 \mathrm{nN}$ of force was applied to the $\mathrm{ZnO}$ nanorods using an AFM probe. In this experiment, the scan speed and feedback gain were set to $0.2 \mathrm{~Hz}$ and 1.0 , respectively. Each measurement was conducted over an area of $16 \mu \mathrm{m} \times 4 \mu \mathrm{m}$, generating $512 \times 64$ data points. Fig. 3(a) presents scatter plots of the current versus the lateral force under different normal force levels. First, the number of data points exceeding lateral force of $5 \mathrm{~V}$ gradually increased from 2 to 94 with an increase in the normal force from $300 \mathrm{nN}$ to $600 \mathrm{nN}$. This implies that the action-reaction forces between the AFM tip and the $\mathrm{ZnO}$ nanorods were more effectively induced by applying a high level of normal force. It should be noted that all lateral force signals were presented in the unit of raw signals, $\mathrm{V}$, because the effect of scan parameters on lateral force signals can be analyzed with raw signals.

If the piezoelectric effect is the only mechanism responsible for current generation, when the AFM tip pushes the stretched side of the $\mathrm{ZnO}$ nanorods, it should induce large lateral force with no current. Thus, current should not be detected when the AFM tip is in contact with the stretched side of the $\mathrm{ZnO}$ nanorods. However, in the scatter plot of $300 \mathrm{nN}$, vanishingly few data points can be observed in the shaded region. This implies that a considerable amount of current was generated via the triboelectric effect associated with the force of friction between the AFM tip and the $\mathrm{ZnO}$ nanorods, even when the normal force was only $300 \mathrm{nN}$. This scatter pattern was not influenced when increasing the normal force to $600 \mathrm{nN}$. It should be also noted that according to the scatter plots in Fig. 3(a) the amount of current originating from triboelectric effect was not distinctly different from that originating from piezoelectric effect. Thus, the use of these scatter plots is constrained to the qualitative identification of the current induced by triboelectric effect between the AFM tip and $\mathrm{ZnO}$ nanorods. Details can be found in the literature [27].

For a force range of $300-600 \mathrm{nN}$, histograms of the CAFM and LFM signals are shown in Fig. 3(b). Using these (a)

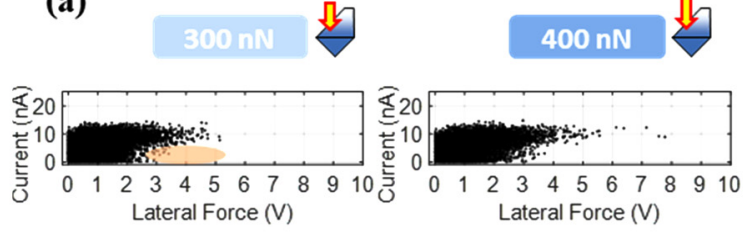

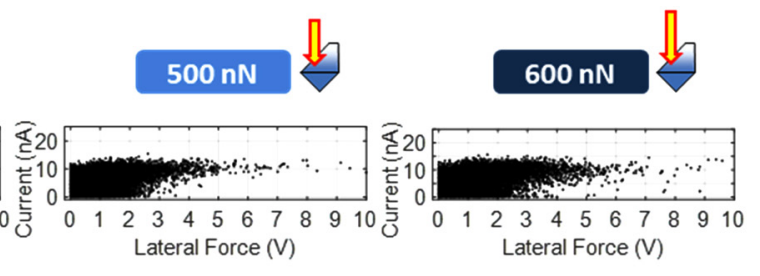

(b)

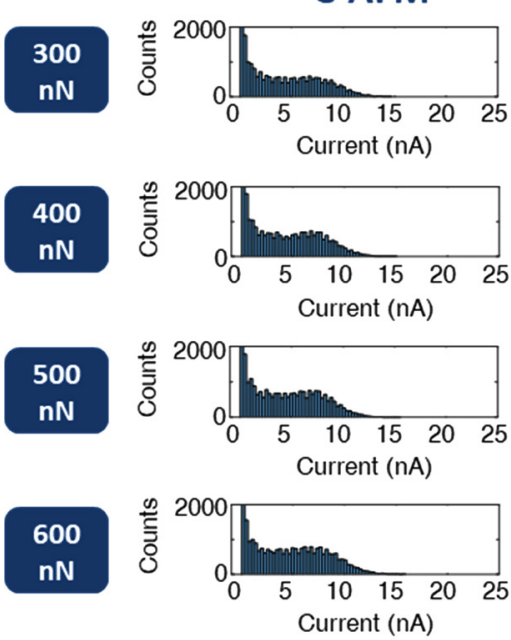

LFM
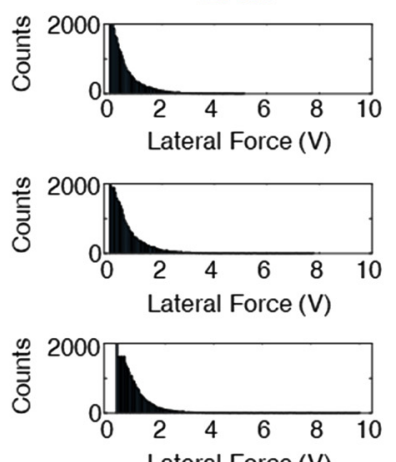

Lateral Force $(V)$

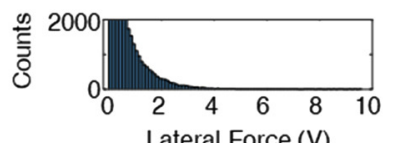

(c)

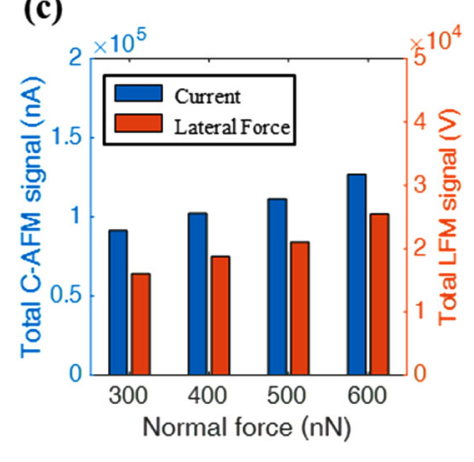

Fig. 3. (a) Scatter plots of the current versus lateral force for various normal force levels, (b) C-AFM and LFM histograms, and (c) the total C-AFM and LFM signals with respect to various amounts of normal force. 
datasets, the total C-AFM and LFM signals can be computed as $\sum_{i=1, j=1}^{i=64, j=512}\left|C-A F M_{i, j}\right|$ and $\sum_{i=1, j=1}^{i=64, j=512} \mathrm{LFM}_{i, j}$, respectively. Fig. 3(c) presents the total C-AFM and LFM signals with respect to different amounts of normal force. The total CAFM and LFM signals in both cases showed steady increases as the normal force was increased. As the normal force was increased from $300 \mathrm{nN}$ to $600 \mathrm{nN}$, the C-AFM signal increased by $38.8 \%$ from $9.14 \times 10^{4} \mathrm{nA}$ to $1.26 \times 10^{5} \mathrm{nA}$ and the LFM signal increased by $58.6 \%$ from $1.60 \times 10^{4}$ to $2.54 \times 10^{4} \mathrm{~V}$. This result indicates that relatively more lateral force was induced when applying higher levels of normal force to the AFM probe, enhancing the deflection of the $\mathrm{ZnO}$ nanorods. The increased current can be attributed to the enhanced piezoelectric effect via the deflection of the $\mathrm{ZnO}$ nanorods and the triboelectric effect from the enhanced force of friction between the AFM tip and the $\mathrm{ZnO}$ nanorods.

Following the result with regard to the different amounts of normal force, the effects of the scan speed on C-AFM and LFM signals were determined, as shown in Fig. 4. It should be noted that in this experiment, the normal force and feedback gain were set to $400 \mathrm{nN}$ and 1.0, respectively. In the experiment, all measurements utilized an area of $16 \mu \mathrm{m} \times 4 \mu \mathrm{m}$, producing $512 \times 64$ data points. Scatter plots of the current versus lateral force at varying scan speeds are shown in Fig. 4(a). First, it could be confirmed that the current originating from the triboelectric effect is considerable regardless of the scan speed. The effect of the scan speed on the C-AFM and LFM signals was rather complex; therefore, the histograms did not show any steady variation of the scatter pattern as the scan speed was increased.

In Fig. 4(b), the maximum current is shown to increase from $18.9 \mathrm{nA}$ to $21.6 \mathrm{nA}$ as the scan speed increases from $0.2 \mathrm{~Hz}$ to $1.1 \mathrm{~Hz}$. However, the counts at $\sim 10 \mathrm{nA}$ decreased considerably when the scan speed exceeded $0.8 \mathrm{~Hz}$. The total C-AFM and LFM signals with respect to the different scan speeds are presented in Fig. 4(c). First, the total LFM signal increased from $1.622 \times 10^{4} \mathrm{~V}$ to $1.686 \times 10^{4} \mathrm{~V}$ as the scan speed was increased from 0.2 to 0.5 . However, when the scan speed exceeded 0.5 , the total LFM signal started to decrease. It is interesting to note that the variation of the total C-AFM signal with respect to the scan speed showed a trend similar to that of the total LFM signal. The maximum total C-AFM
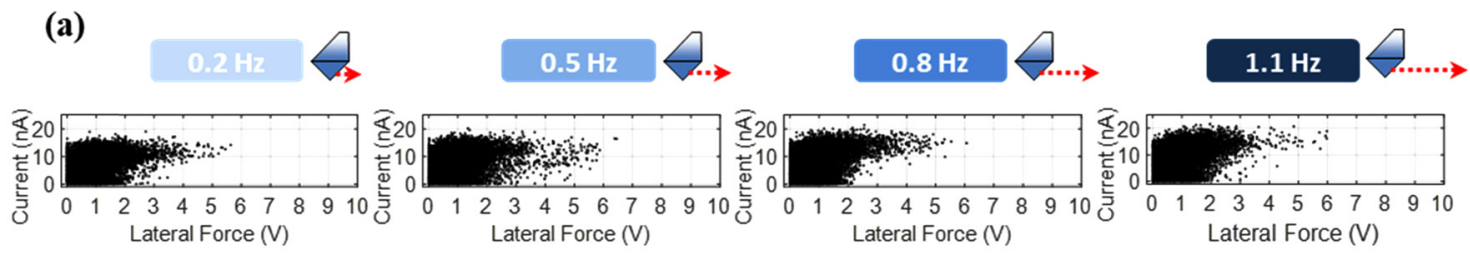

(b)

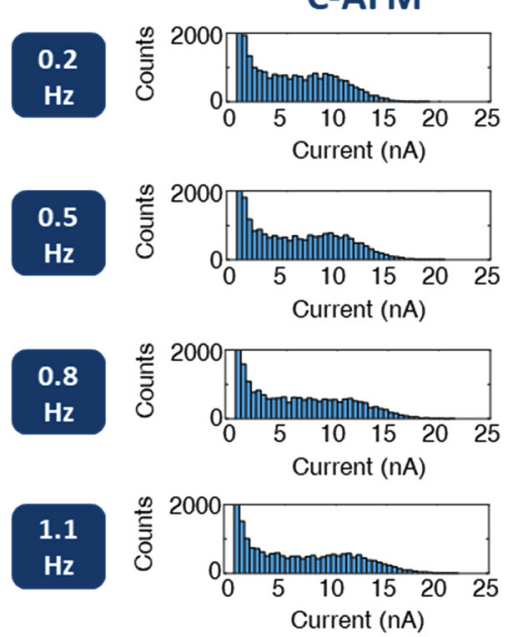

LFM
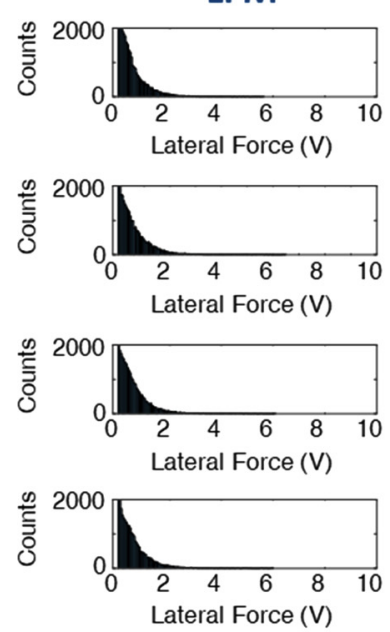

(c)

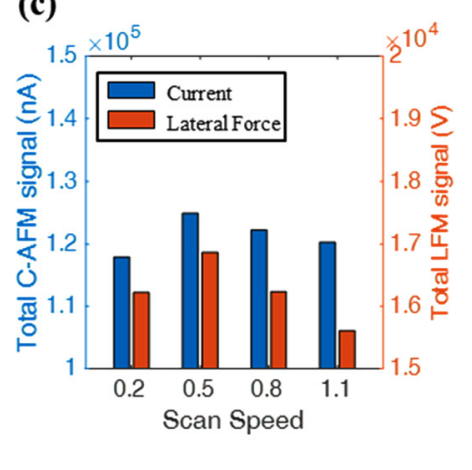

Fig. 4. (a) Scatter plots of the current versus lateral force for various scan speeds, (b) C-AFM and LFM histograms, and (c) total C-AFM and LFM signals with respect to various scan speeds. 


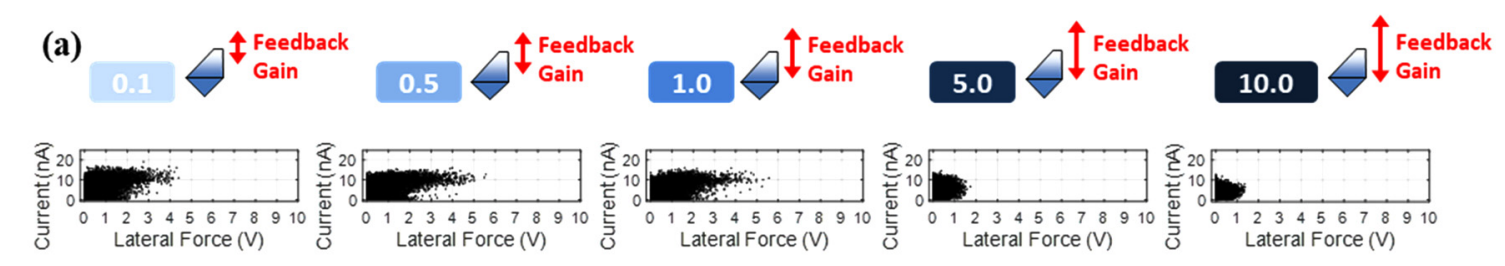

(b)

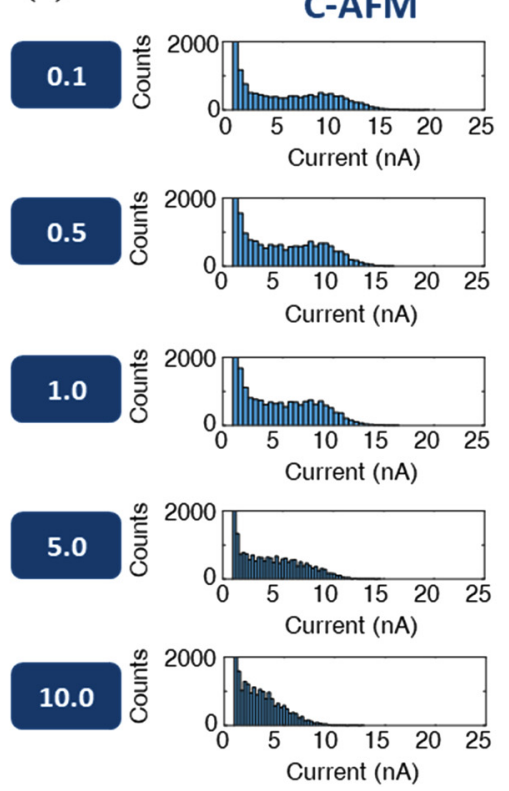

LFM
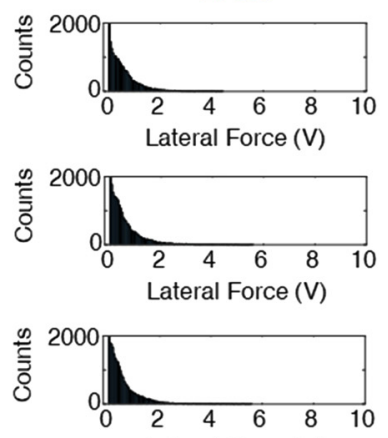

Lateral Force (V)
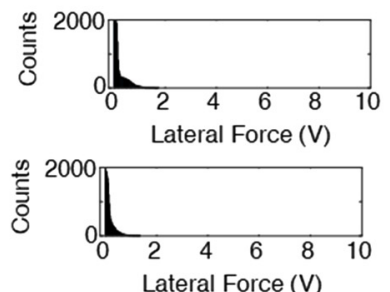

(c)

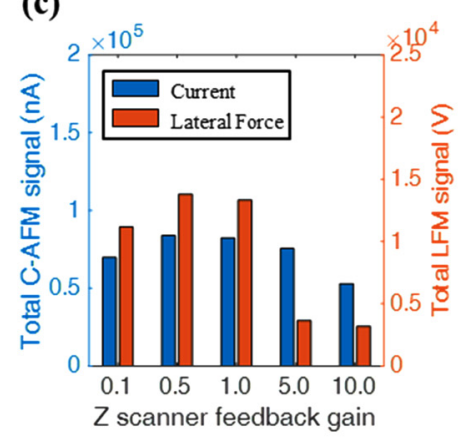

Fig. 5. (a) Scatter plots of the current versus lateral force for various Z scanner feedback gains, (b) C-AFM and LFM histograms, and (c) the total C-AFM and LFM signals with respect to various $Z$ scanner feedback gains.

and LFM signals were generated at a scan speed of 0.5. From this result, the following points can be clarified. C-AFM signals were significantly influenced by LFM signals given that the degree of deflection in $\mathrm{ZnO}$ nanorods and the triboelectricity induced by the force of friction are associated with the amount of lateral force. The mechanisms underlying the variation of the current and lateral force with respect to the scan speed will be explained later in detail with Fig. 6 .

Finally, the effects of the $\mathrm{Z}$ scanner feedback gain on the $\mathrm{C}$-AFM and LFM signals are presented in Fig. 5. The sensitivity of the $\mathrm{Z}$ scanner feedback loop is determined by the gain, meaning that the external force applied to the $\mathrm{ZnO}$ nanorods should be dependent on the magnitude of the gain. It should also be noted that the normal force and scan speed were set to $400 \mathrm{nN}$ and $0.2 \mathrm{~Hz}$, respectively. Measurements at varying gains were conducted using an area of $15 \mu \mathrm{m} \times 3 \mu \mathrm{m}$, generating $512 \times 51$ data points. Fig. $5($ a) presents scatter patterns of the current versus the lateral force at varying $Z$ scanner feedback gains. The typical scatter patterns observed in Fig. 3 and Fig. 4 arise when the gain ranges from 0.1 to 1.0. However, when the gain levels were 5.0 and 10.0, the scatter plots exhibited completely different patterns due to the enhanced sensitivity of the $\mathrm{Z}$ scanner feedback loop.

As shown in the histograms of the LFM signals in Fig. 5(b), the number of instances in which the current exceeded $5 \mathrm{nA}$ increased from 6398 to 7843 as the $\mathrm{Z}$ scanner feedback gain increased from 0.1 to 0.5 . However, when the gain exceeded 1.0, the number of instances in which the current exceeded $5 \mathrm{nA}$ started to decrease. This variation of the trend of the C-AFM signals can be explained by the variation of the LFM signals with respect to the gain. When the gain exceeded 1.0, the $Z$ scanner feedback loop was sensitive enough to reduce the deflection of the $\mathrm{ZnO}$ nanorods sufficiently. As the gain was increased from 0.1 to 0.5 , the 
(a)
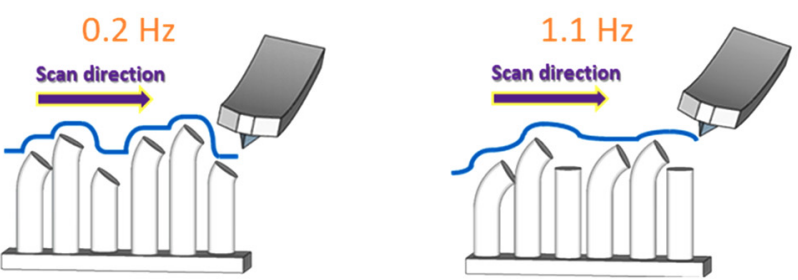

(b)
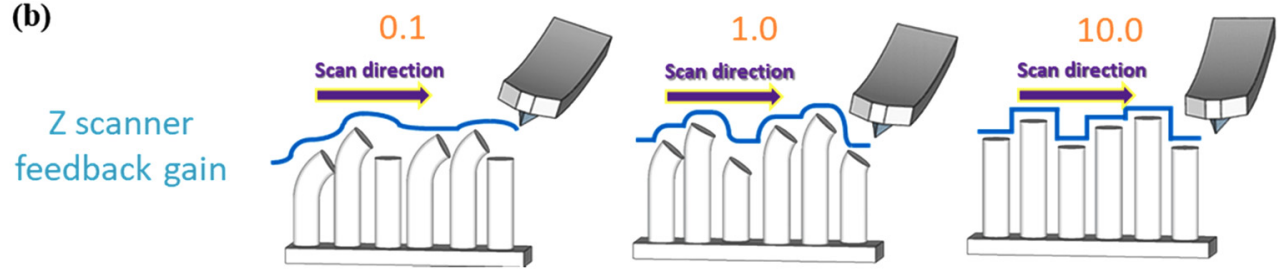

Fig. 6. (a) Schematic illustrating the interactions between the AFM tip and $\mathrm{ZnO}$ nanorods with varying scan speeds, and (b) with varying $\mathrm{Z}$ scanner feedback gains.

total C-AFM and LFM signals also increased, as shown in Fig. 5(c). However, when gain exceeded 1.0, the total CAFM and LFM signals in both cases started to decrease. It should be noted that as gain was increased to 5.0 while the total LFM signal was decreased sharply, the total C-AFM signal decreased gradually. This is explained in detail with the schematic shown in Fig. 6.

Thus far, the effects of the normal force, scan speed, and feedback gain on C-AFM and LFM signals have been discussed. The effects of the normal force on C-AFM and LFM signals were clear, but the interpretation for the C-AFM and LFM signals in response to varying scan speeds or gains requires a systematic approach. To interpret the effects of the scan speed and gain on C-AFM and LFM signals, schematics illustrating the interaction between the AFM tip and $\mathrm{ZnO}$ nanorods were devised, as shown in Fig. 6. First, as depicted in Fig. 6(a), an increase in the scan speed contributed to larger deflection of the $\mathrm{ZnO}$ nanorods, consequently increasing the current. However, when the scan speed exceeded $0.5 \mathrm{~Hz}$, the AFM tip would not touch the short $\mathrm{ZnO}$ nanorods, resulting in reductions of the total C-AFM and LFM signals. It should be noted that as the scan speed was increased from $0.2 \mathrm{~Hz}$ to $1.1 \mathrm{~Hz}$, the total number of instances of current levels exceeding $15 \mathrm{nA}$ gradually increased from 94 to 905 owing to the enhanced deflection of $\mathrm{ZnO}$ nanorods by the high scan speed. Meanwhile, as Fig. 6(b) shows when the gain was increased from 0.1 to 0.5 , the enhanced feedback gain enabled the AFM tip to deflect more $\mathrm{ZnO}$ nanorods, increasing the total C-AFM and LFM signals. However, when the gain was increased to more than 1.0, the $\mathrm{Z}$ scanner feedback loop was too sensitive to deflect $\mathrm{ZnO}$ nanorods, resulting in a considerable reduction in the lateral force. As stated earlier, a large amount of current was still detected at a gain of 10.0 owing to the triboelectric effect.

Topography error signals are directly determined by the $\mathrm{Z}$ scanner feedback gain. It was noted that a positive error signal is generated when the AFM probe climbs a slope, while a negative error signal is generated when the AFM probe comes down a slope. When scanning $\mathrm{ZnO}$ nanorods with the AFM probe, positive error signals should be generated when the AFM tip is in contact with the stretched side of the $\mathrm{ZnO}$ nanorods. Meanwhile, negative error signals should be generated when the AFM tip is released from the $\mathrm{ZnO}$ nanorods.

Fig. 7 shows scatter plots of the current versus the error signal and the lateral force versus the error signal with respect to varying gains. When the gain was 0.1 , the sensitivity of the $\mathrm{Z}$ scanner feedback loop was too low, resulting in a large degree of cantilever deflection. As the feedback gain was increased, the degree of cantilever deflection gradually decreased, reducing positive error signals. This trend was continued as the feedback gain was increased to 10.0. From Fig. 7, it can be seen that the amplitude of the error signals can be controlled by the feedback gain and that the current and lateral force signals are dependent on the error signals. The very small amplitude 


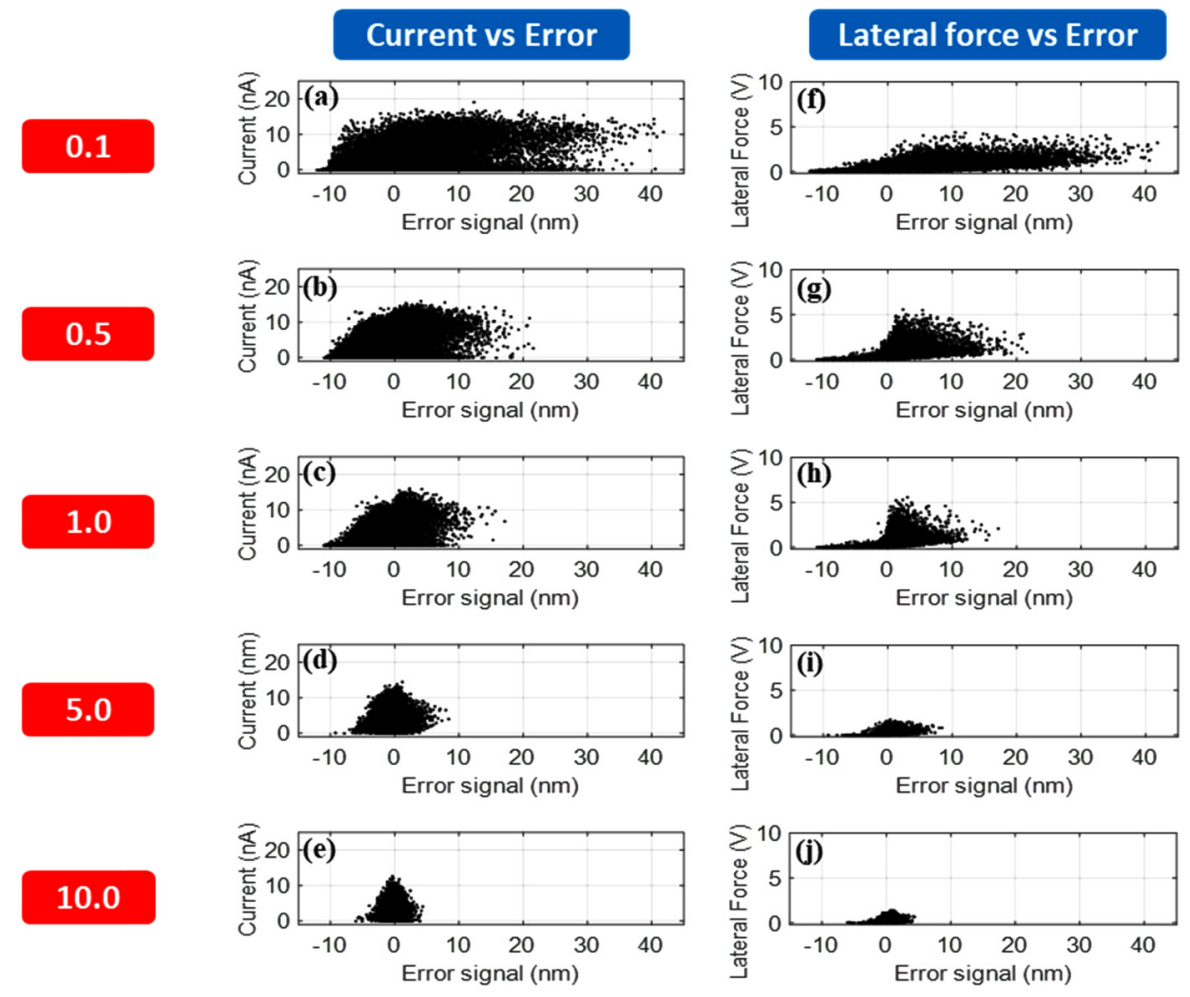

Fig. 7. Scatter plots of the current versus the error signal and the lateral force versus the error signal with respect to various $Z$ scanner feedback gains. (a)-(e) Current versus error signal, and (f)-(j) lateral force versus error signal with varying $Z$ scanner feedback gains from 0.1 to 10.0 .

of the error signal at a gain of 10.0 indicates that the sensitivity of the $\mathrm{Z}$ scanner feedback loop was very high such that deflection of the $\mathrm{ZnO}$ nanorods was scarcely induced by the AFM tip. Consequently, a very small amount of current was produced.

Fig. 8 shows C-AFM, LFM, topography error, and topography images for feedback gains in the range of 0.1 -
10.0. There are several interesting points to be highlighted in Fig. 8. First, C-AFM and LFM images resemble each other when the sensitivity of the $\mathrm{Z}$ scanner feedback loop was low. In this work, large LFM signals were detected when $\mathrm{ZnO}$ nanorods were sufficiently deflected by the AFM tip. However, it is known that C-AFM signals can originate from triboelectricity as well as piezoelectricity. C-AFM signals

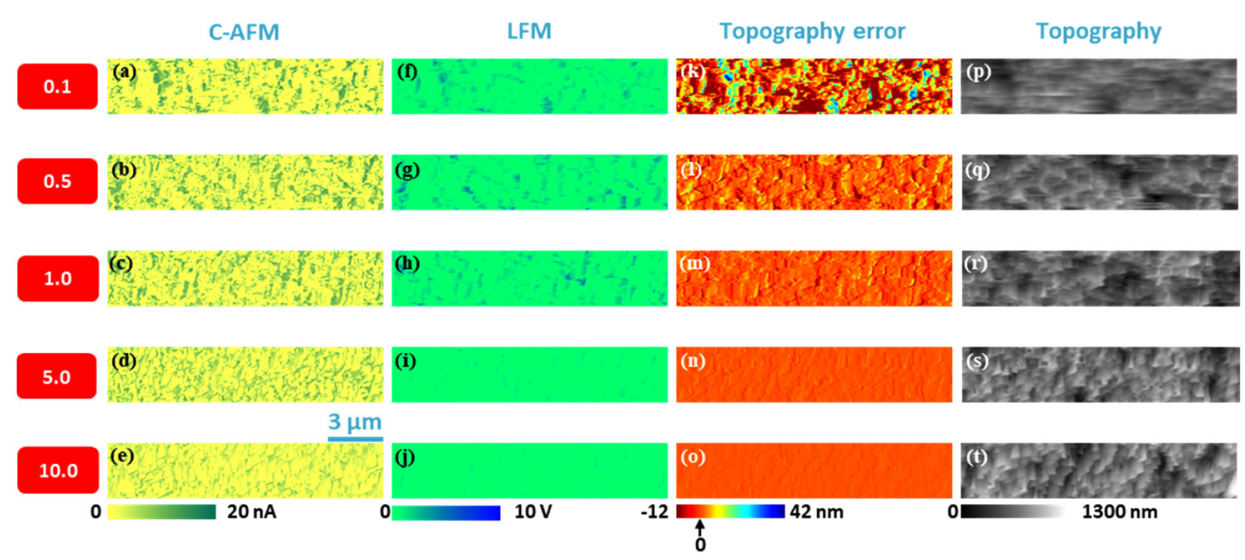

Fig. 8. C-AFM, LFM, topography error, and topography images obtained when the $Z$ scanner feedback gains ranged from 0.1 to 10.0 . (a)(e) C-AFM images, (f)-(j) LFM images, (k)-(o) topography error images, and (p)-(t) topography images with varying $Z$ scanner feedback gains from 0.1 to 10.0 . 
were detected from several places in which no strong LFM signal was detected because the generation of current via triboelectricity does not require large deflection of the $\mathrm{ZnO}$ nanorods. Secondly, when the gain was 0.1 , the $\mathrm{ZnO}$ nanorods were not clearly imaged during the topography assessments. As the feedback gain was increased, individual $\mathrm{ZnO}$ nanorods were more clearly visualized in the topography images. This indicates that free-standing nanomaterials can be clearly visualized by increasing the feedback gain. Thirdly, compared to the C-AFM image at a gain of $0.1, \mathrm{C}$ AFM signals were detected from relatively more $\mathrm{ZnO}$ nanorods when the feedback gain was 1.0. This indicates that the mechanical interaction between the AFM tip and the $\mathrm{ZnO}$ nanorods took place more frequently owing to the enhanced sensitivity of the $\mathrm{Z}$ scanner feedback loop. Finally, when gain was $10.0, \mathrm{ZnO}$ nanorods were not sufficiently deflected by the AFM tip, generating relatively few a LFM signals. In contrast, current signals were still detected from many different places, indicating that these signals originated from triboelectricity.

When varying the scan speed or gain, the same normal force $(400 \mathrm{nN})$ was applied to the AFM probe. The lateral force applied to the $\mathrm{ZnO}$ nanorods by the AFM probe has a magnitude identical to that of the lateral force applied to the AFM probe by $\mathrm{ZnO}$ nanorods. Thus, observing the variation of the total output current/total lateral force in response to changes in the scan speed or gain would provide useful information pertaining to the conversion efficiency of mechanical force when applied to $\mathrm{ZnO}$ nanorods into current. The conversion efficiency of applied lateral force into current can be computed as

$$
\frac{\text { Total output current }}{\text { Total lateral force }}=\frac{\Sigma\left|c-a f m_{i, j}\right|}{\Sigma\left|l f m_{i, j}\right|}
$$

Fig. 9(a) shows a steady increase of the conversion efficiency with an increase in the scan speed. As indicated in Fig. 4(c), both the current and lateral force reached their maximum values when the scan speed was 0.5 . Thereafter, both started to decrease as the scan speed was increased further. However, the decreasing rate of the lateral force was much faster than that of the current as the scan speed was increased. The result in Fig. 9(a) implies that at high scan speed, current can be efficiently generated with a small
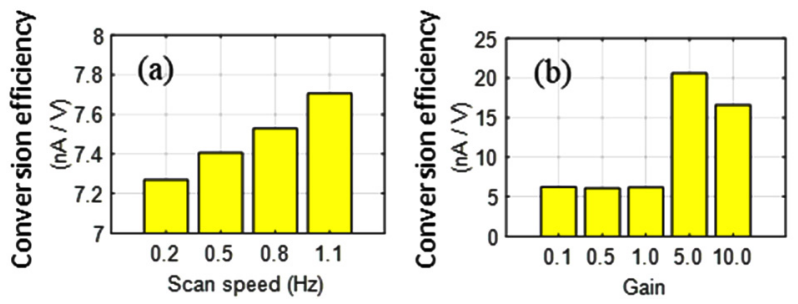

Fig. 9. (a) The conversion efficiency of the applied lateral force into current with respect to different scan speeds, and (b) the Z scanner feedback gain.

amount of externally applied force. Meanwhile, as Fig. 9(b) shows, the highest conversion efficiency was generated when the gain was 5.0, followed by 10.0. In particular, Fig. 5(c) indicates that the lateral force decreased sharply as the gain was increased from 1.0 to 5.0. In contrast, the current only decreased slightly as the gain was changed from 1.0 to 5.0. Such different behaviors of the lateral force and current with respect to different levels of gain can be interpreted as follows. First, the lateral force reduced by the increased feedback gain suppressed the deflection of $\mathrm{ZnO}$ nanorods. However, due to the highly sensitive $Z$ scanner feedback gain, the contact time between the $\mathrm{ZnO}$ nanorods and the AFM tip increased, enhancing the triboelectric effect. Therefore, the result was a moderate decrease of the current as the gain was increased. Meanwhile, it should be noted that the conversion efficiency of mechanical energy into electrical energy cannot be directly obtained via Eq. (1) because the vertical displacement of the AFM tip is not provided from this experimental approach.

Based on the present study, the optimal scan parameters for $\mathrm{C}$-AFM analysis on $\mathrm{ZnO}$ nanorods can be proposed as follows. Because it is desirable to induce the sufficient amount of current via deflection of $\mathrm{ZnO}$ nanorods, scan speed of $0.5 \mathrm{~Hz}$ and $\mathrm{Z}$ scanner feedback gain of 0.5-1.0 can be the optimal scan conditions. In the case of normal force, too large normal force could damage the AFM tip and $\mathrm{ZnO}$ nanorods. Therefore, the minimum normal force producing sufficient amount of C-AFM signal would be desirable.

\section{Conclusion}

For vertically grown $\mathrm{ZnO}$ nanorods, the dependency of CAFM signals on certain scan parameters, in this case the 
normal force, scan speed, and Z scanner feedback gain, was investigated. In this work, for a systematic analysis, C-AFM and LFM signals were simultaneously acquired during the measurements. First, as the normal force was increased, the total LFM signal increased through the enhanced deflection of $\mathrm{ZnO}$ nanorods, increasing the total C-AFM signal. From scatter plots, it was confirmed that the current originated from triboelectricity associated with the friction between the $\mathrm{ZnO}$ nanorods and the AFM tip as well as piezoelectricity with regard to the deflection of the $\mathrm{ZnO}$ nanorods. As the scan speed was increased to $0.5 \mathrm{~Hz}$, both the total LFM signal and the total C-AFM signal increased due to the increased degree of deflection in the $\mathrm{ZnO}$ nanorods. However, when the scan speed exceeded $0.5 \mathrm{~Hz}$, both the total LFM signal and the total C-AFM signal started to decrease. It was speculated that when the scan speed was too high, the AFM tip would not have been able to deflect short $\mathrm{ZnO}$ nanorods. Finally, as the feedback gain was increased from 0.1 to 0.5 , the total LFM signal and the total C-AFM signal increased. The enhanced sensitivity of the $\mathrm{Z}$ scanner feedback loop enabled the AFM tip to deflect more $\mathrm{ZnO}$ nanorods, generating a larger amount of current. However, when the gain exceeded 1.0, the $\mathrm{Z}$ scanner feedback loop was too sensitive to deflect $\mathrm{ZnO}$ nanorods, reducing the total LFM signal. In contrast, the total C-AFM signal showed only a moderate decrease owing to the enhanced triboelectric effect via the increased contact time between the AFM tip and the $\mathrm{ZnO}$ nanorods. Finally, when acquiring topography images of free-standing nanorods, the deflections of the nanorods were minimized by increasing the sensitivity of the $\mathrm{Z}$ scanner feedback loop, producing a clear view of individual nanorods.

\section{Acknowledgement}

This study was supported by the Research Program funded by the SeoulTech(Seoul National University of Science and Technology). We would like to thank Prof. Il-Kyu Park at Seoul National University of Science and Technology for his help with growing $\mathrm{ZnO}$ nanorods.

\section{REFERENCES}

1. Z. L. Wang and W. Wu, Angew. Chem. Int. Ed. 51, 11700 (2012).

2. W. Guo, C. Tan, K. Shi, J. Li, X. X. Wang, B. Sun, X. Huang, Y. Z. Long, and P. Jiang, Nanoscale 10, 17751 (2018).

3. Y. Yang, H. Pan, G. Xie, Y. Jiang, C. Chen, Y. Su, Y. Wang, and H. Tai, Sens. Actuators A Phys. 301, 111789 (2020).

4. X. Chen, K. Parida, J. Wang, J. Xiong, M. F. Lin, J. Shao, and P. S. Lee, ACS Appl. Mater. Interfaces 9, 42200 (2017).

5. C. Li, W. Luo, X. Liu, D. Xu, and K. He, Nanomaterials 6 , 1 (2016).

6. J. Yan and Y. G. Jeong, Compos. Sci. Technol. 144, 1 (2017).

7. X. Chen, X. Li, J. Shao, N. An, H. Tian, C. Wang, T. Han, L. Wang, and B. Lu, Small 13, 1604245 (2017).

8. D. Berlincourt and H. Jaffe, Phys. Rev. 111, 143 (1958).

9. C. Wan and C. R. Bowen, J. Mater. Chem. A 5, 3091 (2017).

10. T. R. Shrout and S. J. Zhang, J. Electroceramics 19, 111 (2007).

11. L. Schmidt-Mende and J. L. MacManus-Driscoll, Mater. Today 10, 40 (2007).

12. Z. L. Wang, J. Phys.: Condens. Matter 16, R829 (2004).

13. H. Rotella, Y. Mazel, S. Brochen, A. Valla, A. Pautrat, C. Licitra, N. Rochat, C. Sabbione, G. Rodriguez, and E. Nolot, J. Phys. D: Appl. Phys. 50, 485106 (2017).

14. L. Xu, J. Miao, Y. Chen, J. Su, M. Yang, L. Zhang, L. Zhao, and S. Ding, Optik 170, 484 (2018).

15. Y. C. Yang, C. Song, X. H. Wang, F. Zeng, and F. Pan, Appl. Phys. Lett. 92, 012907 (2008).

16. A. T. Le, M. Ahmadipour, and S.Y. Pung, J. Alloys Compd. 844, 156172 (2020).

17. Z. L. Wang and J. Song, Science 312, 242 (2006).

18. J. Song, J. Zhou, and Z. L. Wang, Nano Lett. 6, 1656 (2006).

19. M. P. Lu, J. Song, M. Y. Lu, M. T. Chen, Y. Gao, L. J. Chen, and Z. L. Wang, Nano Lett. 9, 1223 (2009).

20. C. Y. Chen, T. H. Liu, Y. Zhou, Y. Zhang, Y. L. Chueh, Y. H. Chu, J. H. He, and Z. L. Wang, Nano Energy 1, 424 (2012).

21. M. Y. Lu, J. Song, M. P. Lu, C. Y. Lee, L. J. Chen, and Z. L. Wang, ACS Nano 3, 357 (2009).

22. Y. S. Zhou, K. Wang, W. Han, S. C. Rai, Y. Zhang, Y. Ding, C. Pan, F. Zhang, W. Zhou, and Z. L. Wang, ACS Nano 6, 6478 (2012). 
23. C. T. Huang, J. Song, C. M. Tsai, W. F. Lee, D. H. Lien, Z. Gao, Y. Hao, L. J. Chen, and Z. L. Wang, Adv. Mater. 22 , 4008 (2010).

24. Y. F. Lin, J. Song, Y. Ding, S. Y. Lu, and Z. L. Wang, Appl. Phys. Lett. 92, 022105 (2008).

25. C. Y. Chen, G. Zhu, Y. Hu, J. W. Yu, J. Song, K. Y. Cheng, L. H. Peng, L. J. Chou, and Z. L. Wang, ACS Nano 6, 5687 (2012).

26. C. Wen, X. Jing, F. F. Hitzel, C. Pan, G. Benstetter, and M. Lanza, ACS Appl. Mater. Interfaces 11, 15183 (2019).

27. Y. Yang and K. Kim, Sci. Rep. 11, 2904 (2021).
28. D. Sobola, Ş. Ţălu, S. Solaymani, and L. Grmela, Microsc. Res. Tech. 80, 1328 (2017).

29. B. Xue, Y. Yan, Z. Hu, and X. Zhao, Scanning 36, 263 (2014).

30. G. Li, B. Mao, F. Lan, and L. Liu, Rev. Sci. Instrum. 83, 113701 (2012).

31. F. J. Giessibl, Rev. Mod. Phys. 75, 949 (2003).

32. H. Yan, X. Gong, J. Chen, and M. Cheng, Met. Mater. Int. 27, 2249 (2021).

33. D. Park, Y. Yang, and K. Kim, Korean J. Met. Mater. 59, 209 (2021). 